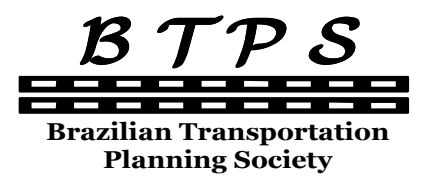

Journal of Transport Literature

Vol. 8, n. 1, pp. 285-303, Jan. 2014

Research Directory
JTL | RELIT

www.transport-literature.org

\title{
Simulação do ruído sonoro no entorno do aeroporto de Ribeirão Preto
}

[Simulation of aircraft noise in the surroundings of the Ribeirão Preto airport]

\author{
Antônio Carlos Dinato*, Ricardo Ernesto Schaal
}

Departamento Aeroviário Do Estado De São Paulo - Brazil, EESC-USP - Brazil

Submitted 3 Jul 2012; received in revised form 25 Jan 2013; accepted 28 Jan 2013

\begin{abstract}
Resumo
Este artigo apresenta simulações de curvas de ruído utilizando o programa INM (Integrated Noise Model) e Transcad com o objetivo de verificar as áreas onde a população que reside nas vizinhanças do aeroporto é afetada pelo aumento do tráfego aéreo e mudanças climáticas. Este estudo foi motivado pelas reclamações junto ao Ministério Público pela população residente no entorno do aeroporto. Os resultados mostram variações significativas na população afetada com a mudança da posição das curvas isofônicas entre 55 e $65 \mathrm{~dB}(\mathrm{~A})$ e menos significativas acima de $65 \mathrm{~dB}(\mathrm{~A})$. Entende-se que simulações permitem estimar ordem de grandeza do impacto das aeronaves nas vizinhanças do aeroporto norteando futuros levantamentos de dados reais. As divergências de uma simulação com valores reais podem ser geradas pelo não seguimento dos padrões estabelecidos nas cartas de voo, ventos de direções diversas e a não inclusão do relevo da região. Os resultados mostraram que modificações nos procedimentos de pouso e decolagem podem mitigar a poluição sonora.
\end{abstract}

Palavras-Chave: simulação com INM, população afetada pelo ruído.

\begin{abstract}
This article presents simulations of curves of aircraft noise using the INM (Integrated Noise Model) program as well as the Transcad, in order to identify the areas in the surroundings of the airport where the population is more affected by the increase of the aircraft traffic and the climate changes. This study was initiated after the complaints made by the population living in the surroundings of the airport and received by the Prosecuting Office. The overall results indicated substantial variations in the population affected by the changes in the position of the isophonic curves between 55 and $65 \mathrm{~dB}(\mathrm{~A})$ and a less expressive effect above $65 \mathrm{~dB}(\mathrm{~A})$. Simulations like those performed here allow one to estimate the magnitude order of the impact of aircrafts in the surroundings of the airport, guiding future data collections in the field. Discrepancies between the predicted data and the field data can be caused by noncompliance to the standards established by the flight charts, by winds of different directions and the disregard of the surrounding relief. The results showed that modifications to the landing and takeoff procedures can mitigate noise pollution.
\end{abstract}

Key words: simulation with INM, population affected by noise.

*Email: dinato@gmail.com.

\section{Recommended Citation}

Dinato, A. C. and Schaal, R. E. (2014) Simulação do ruído sonoro no entorno do aeroporto de Ribeirão Preto. Journal of Transport Literature, vol. 8, n. 1, pp. 285-303.

- JTL/RELIT is a fully electronic, peer-reviewed, open access, international journal focused on emerging transport markets and published by BPTS - Brazilian Transport Planning Society. Website www.transport-literature.org. ISSN 2238-1031.

This paper is downloadable at www.transport-literature.org/open-access. 


\section{Introdução}

Este artigo apresenta simulações de curvas de ruído utilizando o programa INM (Integrated Noise Model) e Transcad com o objetivo de verificar as áreas onde a população que reside nas vizinhanças do Aeroporto de Ribeirão Preto é afetada pelo aumento do tráfego aéreo e mudanças climáticas. Este estudo foi motivado pelas reclamações junto ao Ministério Público pela população residente no entorno do aeroporto. Entende-se que simulações permitem estimar ordem de grandeza do impacto das aeronaves nas vizinhanças do aeroporto norteando futuros levantamentos de dados reais.

A preocupação dos organismos internacionais de aviação civil a respeito do impacto da aviação no meio ambiente teve seu marco inicial em 1944, durante a Convenção de Aviação Civil Internacional de Chicago. O impacto ambiental causado pela aviação civil está ligado diretamente aos problemas decorrentes da operação das aeronaves e à construção e operação dos aeroportos. Os problemas mais relevantes são os ruídos aeronáuticos, a emissão de gases provenientes da queima de combustível, os resíduos sólidos e líquidos, danos à flora e fauna e o uso/interferência nos recursos naturais citados também por Horonjeff \& McKelvey (1993).

Berglund et al. (1999) descrevem o ruído que a comunidade percebe como: ruído ambiental, doméstico e residencial. As fontes causadoras podem ser: rodoviário, ferroviário, aeronáutico, indústrias, construção civil, serviços públicos, vizinhanças etc.

A preocupação com o ruído tem levado alguns países a considerar a possibilidade de proibir a operação de certas aeronaves ruidosas em seus aeroportos. No entanto, as restrições deste tipo podem ter consequências econômicas significativas para as empresas aéreas, tanto para as estabelecidas nos países que adotam essas medidas, como as estabelecidas em outros países, particularmente nos países desenvolvidos, que prestam serviços, direta ou indiretamente, na fabricação de aeronaves. As empresas aéreas terão que trocar suas aeronaves por outras mais novas ou recondicionadas com motores mais silenciosos, e com equipamento de atenuação de ruído, ou transferir as aeronaves mais ruidosas para outras rotas (Icao, 2004).

Trabalhos científicos relacionados com o ruído ambiental demonstram que uma pessoa só consegue relaxar totalmente durante o sono em níveis de ruído abaixo de $39 \mathrm{~dB}(\mathrm{~A})$, enquanto 
a Organização Mundial de Saúde estabelece 55 dB(A) como nível médio de ruído diário para uma pessoa viver bem. Acima de $75 \mathrm{~dB}(\mathrm{~A})$ começa a acontecer o desconforto acústico, ou seja, para qualquer situação ou atividade, o ruído passa a ser um agente de desconforto. Nessas condições há uma perda da inteligibilidade da linguagem, a comunicação fica prejudicada, passando a ocorrer distrações, irritabilidade e diminuição da produtividade no trabalho. Acima de $80 \mathrm{~dB}(\mathrm{~A})$, as pessoas mais sensíveis podem sofrer perda de audição, o que se generaliza para níveis acima de $85 \mathrm{~dB}(\mathrm{~A})$ (Fernandes, 2002).

Os níveis de ruído e a máxima exposição diária permissível estão descritas na ABNT - NBR 11415 (ABNT, 1990), onde o tempo máximo de exposição para um ruído de $85 \mathrm{~dB}(\mathrm{~A})$ é de no máximo 8 horas diárias e para um ruído de $115 \mathrm{~dB}(\mathrm{~A})$ o máximo é de 7 minutos. De acordo com Dani e Garavelli (2001) em especial, o ruído urbano resulta em comprometimento à qualidade de vida das pessoas, produzindo problemas fisiológicos de saúde, interferências na comunicação, estresse, irritabilidade, sensação de incômodo, perda auditiva total entre outros. Além disso, o diagnóstico ambiental sonoro não pode ser limitado unicamente à medida de parâmetros objetivos. Existem variáveis subjetivas que influenciam na resposta da população do entorno do aeroporto há um tempo (segundos) de exposição sonora. As variáveis não estritamente acústicas são aquelas que interferem nos caminhos de propagação do som, modificando a sua percepção no local de recepção (Vallet, 2001). As variáveis subjetivas decorrem do incômodo, do medo da queda de aeronaves, da desvalorização de imóveis, de que as autoridades não estejam preocupadas com a situação e de que a situação acústica venha ser modificada, passando a um estágio insuportável (Macedo, 2004).

A Organização Mundial de Saúde (WHO, 2010) preconiza que a poluição sonora é hoje, depois da poluição da água, o problema ambiental que afeta o maior número de pessoas no mundo. Sousa (2004) menciona que no Brasil, a avaliação de impacto ambiental sonoro passou a ser exigido como parte integrante dos Estudos Prévios de Impacto Ambiental (EPIA) e como instrumento de execução da Política Nacional de Meio Ambiente (PNMA).

Segundo Nogueira e Slama (2005) como ocorre em outros países, no Brasil o ruído de um aeroporto é uma questão que tem que ser discutida, e necessita de definição de zonas de ruído ideais, no entanto, é um desafio. Um dos principais problemas ao lidar com o zoneamento do ruído em áreas vizinhas, é o de como quantificar esse impacto na formulação de políticas de controle do ruído. 
A ausência de um planejamento adequado da ocupação do entorno do aeródromo irá provocar, a médio e em longo prazo, um conflito previsível entre essa ocupação e a atividade aeronáutica, Damião e Rocha (1998).

Nos EUA, os estudos com o ruído aeronáutico em aeroportos civis iniciaram-se na década de 60, quando as comunidades afetadas pressionaram as autoridades aeroportuárias a estabelecer restrições com relação ao ruído (ICAO, 1970). A FAA - Federal Aviation Administration, através da publicação "Noise Abatement Policy of 1976", criou a lei que determinou a Política de Redução de Ruído da Aviação e as responsabilidades compartilhadas para a redução do ruído aeronáutico ao redor dos aeroportos. Após este período, surgiram leis regulamentando fatores ligados ao ruído, que foram as seguintes: Quiet Communities Act of 1978 and the Aviation Safety and Noise Abatement Act of 1979 (Werlich and Krinsky, 1981). Atualmente nos Estados Unidos a lei federal que forma a base para o controle do ruído aeronáutico e o programa de compatibilidade de uso do solo é a elaborada pela Federal Aviation Regulation, FAR Part 150 - Noise Compatibility Program (FAA, 2004).

O trabalho está assim organizado: primeiro, na Seção 1, serão apresentadas definições e conceitos do ruído aeronáutico. Em seguida, na Seção 2, são discutidos parâmetros de avaliação da pressão sonora. Na Seção 3 é apresentada a área de estudo e na Seção 4, feita as análise e discussão dos resultados. Por fim, são apresentadas as conclusões.

\section{O ruído e seus conceitos}

Em 1969 a NEPA - National Environmental Policy Act começou a discutir ruídos e suas definições. Em 1971, com o Anexo XVI da ICAO - International Civil Aviation Organization, definido no Brasil como OACI - Organização da Aviação Civil Internacional, com o titulo de Environmental Protection, Volume I - Aircraft Noise (1993) foi instituído o primeiro conjunto de normas e práticas recomendadas sobre o ruído de aeronaves. Neste documento figuram todas as disposições relativas aos aspectos da aviação ligados ao ambiente, à certificação acústica de aeronaves (grandes aeronaves subsônicas a jato, aeronaves a pistão e helicópteros) e aos métodos para medir e monitorar o ruído. 
Em 2001, uma assembleia da ICAO aprovou o conceito de abordagem equilibrada (balanced approach) para a gestão do ruído aeronáutico (Apêndice C da Assembly Resolution A33-7). Esta abordagem identifica o problema do ruído aeroportuário e analisa as várias medidas úteis que visam reduzir o ruído através de quatro variáveis: Redução da fonte (aeronaves mais silenciosas); Planejamento e gestão do uso do solo no entorno do aeroporto; Redução de ruído através de procedimentos operacionais; e Redução de ruído mediante restrição de operação.

Para se conhecer o ruído ambiental no entorno de aeroportos implica em desenvolver programas para identificar os elementos que compõem o sistema aeroportuário; delinear no solo, de forma clara, os locais de mesma intensidade de ruído, constituindo por mapas com as curvas isofônicas, gerados por um modelo ou por medidas de campo.

A partir deste conhecimento será possível fornecer subsídios aos órgãos reguladores que poderão acionar ações mitigadoras.

Macedo (2004) menciona que os estudos do ruído aeroportuário envolvem procedimentos considerados não triviais, devendo-se observar diversos elementos (direção e velocidade do vento, temperatura, pressão, etc..) que interagem entre si e cujas características estão sempre em transformação dinâmica.

Slama et al. apud Revoredo (2011), cita que além da sensação de incomodo, o ruído aeroportuário produz muitos efeitos adversos dos quais os mais importantes são a interferência na comunicação durante o dia e o distúrbio do sono durante a noite. O ruído urbano produz efeitos semelhantes. Baseado nestas características, a regulamentação para zoneamento urbano adota critérios diferenciados para avaliar os ruídos diurno e noturno. A métrica geralmente adotada é o nível sonoro equivalente. Para que seja possível determinar o nível sonoro em um medidor utilizando esta métrica é necessário definir um período de avaliação o qual estará sujeito às características temporais do ruído considerado. 


\section{Parâmetros de avaliação da pressão sonora}

A métrica mais utilizada ao longo das ultimas décadas em avaliações de ruído aeroportuário é denominada nível sonoro equivalente dia e noite (do inglês, day night average sound level) e denotada por $L_{d n}$ (ou DNL). Sua utilização é baseada na ideia de avaliar o número de reclamações geradas na vizinhança do aeroporto em função de sua operação.

O $L_{d n}$ é considerado por muitos como a melhor métrica para medição de ruído que pode ser uniformemente aplicada na medição em comunidades e em torno de aeroportos, e para a qual existe uma relação altamente confiável entre o ruído emitido e a reação das pessoas.

O $L_{d n}$ leva em consideração que o som é uma fonte de energia e, para se avaliar os danos causados à audição, deve-se considerar não apenas o seu nível, mas também seu tempo de duração. Considera a magnitude do ruído de todos os eventos sonoros ocorridos durante um período de 24 horas, o número de eventos, e a alta sensibilidade ao ruído durante períodos típicos de descanso.

Os níveis de ruído que ocorrem durante o período noturno das $22 \mathrm{~h} 00 \mathrm{~min}$ às $07 \mathrm{~h} 00 \mathrm{~min}$, são dadas uma penalidade de $10 \mathrm{~dB}$ de ponderação de tal forma que os cálculos do $L_{d n}$ fica como o apresentado na Expressão (1):

$$
L_{d n}=10 \log \left\{\frac{1}{24}\left[\frac{1}{3600}\left(\sum_{d=1}^{N D} 10^{\frac{L A d}{2}} \frac{d}{2}\right)+\frac{1}{360}\left(\sum_{n=1}^{N N} 10^{\frac{L A n}{10}} \frac{n}{2}\right)\right]\right\}
$$

Onde:

$N D$ = número total de sobrevoos ocorridos durante o período diurno;

$N N$ = número total de sobrevoos ocorridos no período noturno;

$d$ = duração de cada evento diurno;

$n$ = duração de cada evento noturno;

$L A d$ = nível máximo de ruído em $\mathrm{dB}(\mathrm{A})$ dos sobrevoos diurno;

$L A n$ = nível máximo de ruído em $\mathrm{dB}(\mathrm{A})$ dos sobrevoos noturno. 
A média anual diária definida será a que melhor represente um dia típico do aeroporto. Nesta média inclui-se a condição do número e do tipo de operação, a rota das operações, configuração das pistas, peso da aeronave, temperatura e vento.

O Airport Planning Manual - DOC-9184 - Parte 2 (ICAO, 2002), relaciona os valores de $L_{d n}$ em $\mathrm{dB}(\mathrm{A})$ e a reação esperada da comunidade no seu entorno, definindo áreas em função dos níveis de $L_{d n}$.

Valor do $L_{d n}-\mathbf{d B}(\mathbf{A})$

Menor que 65 - Área III

Entre 65 e 75 - Área II

Maior que 75 - Área I

\section{Reação das comunidades}

Nenhuma reclamação é esperada. Ambiente pouco ruidoso. Ambiente medianamente ruidoso. É esperado grande volume de reclamações por parte dos residentes.

Ambiente extremamente ruidoso. São esperadas reclamações generalizadas por parte dos residentes. É possível ação comunitária contra o aeroporto.

Existem outras métricas para a determinação do ruído as quais são especificadas abaixo:

O nível sonoro equivalente (do inglês, equivalent sound level), $L_{A e q d B(A)}$, é uma métrica cumulativa que determina o nível sonoro em estado estacionário em um determinado intervalo de tempo. Esta métrica é apropriada para a avaliação de ruído de aeronaves quando o mesmo afeta atividades com períodos menores do que 24 horas de duração, Expressão 2.

$$
L_{\text {Aeq }}=10 \log _{10}\left[\frac{1}{T} \int_{0}^{T} 10 \frac{L_{A}(t)}{10} d t\right]
$$

O nível de exposição sonora SEL (do inglês, sound exposure level), (também representado pela sigla $L_{A E}$ ), representa a intensidade e a duração de um som. Ele é a expressão logarítmica da energia sonora associada a um determinado ruído em um local específico. Eventos sonoros individuais variantes no tempo, tais como sobrevoos de aeronaves, tem duas características principais: um nível sonoro que é modificado ao longo do evento e um período de tempo ao longo do qual o som é percebido. O SEL prove a medição do impacto do evento 
como um todo, porém não representa diretamente o nível sonoro que é ouvido em um instante de tempo qualquer como mostrado na Expressão 3.

$$
S E L=10 \log _{10}\left[\frac{1}{T_{0}} \int_{0}^{T} 10 \frac{L(t)}{10} d t\right]
$$

No estudo proposto o cálculo do $L_{d n}$ foi realizado utilizando-se da equação dada pela norma SAE AIR 1845:1986 que é a norma utilizada pelo INM para a determinação do $L_{d n}$, na qual se utiliza os valores do SEL $\left(L_{A E}\right)$ de todas as operações aeronáuticas do período da medição de campo. Deve-se considerar o monitoramento em diversos pontos com tempo mais curtos de medição, porém com uma gama maior da operação dessas aeronaves.

\section{3. Área de estudo}

O Aeroporto Estadual de Ribeirão Preto - SBRP (código ICAO - International Civil Aviation Organization) está situado a $7 \mathrm{~km}$ do centro da cidade com pista de pouso e decolagem de $2100 \times 45 \mathrm{~m}$, altitude de $550 \mathrm{~m}$, coordenadas geodésicas definidas pelo ICA (Instituto de Cartografia da Aeronáutica) $21^{\circ} 28^{\prime} 11^{\prime}$ 'S e $47^{\circ} 46^{\prime} 36^{\prime}$ 'W. Sua área patrimonial é de 161,52 ha. O aeroporto de Ribeirão Preto tem uma média anual de $29^{\circ} \mathrm{C}$ de temperatura e ventos que giram em torno de $8 \mathrm{kt}(15 \mathrm{~km} / \mathrm{h})$.

Para este estudo foi utilizado o programa INM 6.0 (Integrated Noise Model) com o qual se realiza simulações de cálculos das curvas de ruído em duas situações:

1. Padrão e,

2. Com aumento da temperatura e da gradiente do vento.

As curvas do estudo proposto são a de $55 \mathrm{~dB}(\mathrm{~A}), 65 \mathrm{~dB}(\mathrm{~A})$ e $75 \mathrm{~dB}(\mathrm{~A})$.

Os contornos de curvas de igual nível de ruído constituem o resultado gráfico fundamental do processo de cálculo e podem ser incorporados aos mapas de compatibilidade de uso do solo como também servirem para o planejamento e direcionamento do plano diretor do município. 
Com esta métrica se consegue identificar a compatibilidade do aeroporto com a ocupação no seu entorno. Estes procedimentos auxiliam na adoção de medidas mitigadoras que visam controlar o ruído ambiental.

Neste estudo foi feito um comparativo entre as operações que eram realizadas diariamente no aeroporto antes da entrada em operação de uma nova empresa, o que acarretou um aumento de mais 24 operações de pouso e decolagem por dia no aeroporto de Ribeirão Preto. A aeronave em operação é o Boeing 737-300.

\subsection{Dados para a simulação no INM}

O programa INM utilizado para a simulação para determinar as curvas de ruído emitido por um avião leva em consideração os seguintes fatores: peso, a potência do motor, a reversão do fluxo dos motores, a rolagem dos pneus durante a aterrissagem e o ruído emitido durante os testes de certificação. Também considera as condições meteorológicas (velocidade do vento, umidade do ar, variação da temperatura com a altitude, assim como a natureza do solo) (FAA, 1983).

A rota do avião durante o procedimento de pouso e decolagem é informação fundamental para simulação. A rota é uma sequência de segmentos, identificados no espaço por suas coordenadas geodésicas, marcada nas cartas publicadas pelo DECEA (Departamento de Controle do Espaço Aéreo).

Abaixo se apresenta dados necessários para alimentar o programa INM que irá gerar curvas de ruído nas distintas condições:

a) Informação do aeroporto

- A latitude e longitude é o ponto de referencia do aeroporto e pode ser obtida nas cartas do ICA (Instituto de Cartografia da Aeronáutica).

- As posições das pistas e altitude podem ser encontradas nas publicações do DECEA.

- A temperatura, velocidade do vento, umidade relativa e pressão barométrica devem ser obtidas diretamente no campo através de uma estação meteorológica. 
b) Informação sobre a aeronave

- Tipo da aeronave, motor, peso, configuração e potencia do motor. Como o programa INM contém os dados em seu banco, não há necessidade de obtê-los nos manuais dos fabricantes das aeronaves.

- Número de movimentos, tipo de operação e trajetórias dos voos são obtidos nos HOTRANS (Horários de Transporte) e nas publicações do DECEA.

c) Composição do cálculo do ruído

- Geometria da pista, que no caso foi definida com as coordenadas geodésicas, determinadas por meio de receptor GPS.

- Os dados da população foram obtidos pelo IBGE (Instituto Brasileiro de Geografia e Estatística) (2000). A planta obtida da Prefeitura de Ribeirão Preto foi georreferenciada para obtermos um resultado preciso quanto à localização destes setores censitários.

\subsection{Simulações do cenário - procedimentos empregados}

Independentemente de como se define o conjunto de operações, o importante é que se tenham todas as operações relevantes que representem da melhor forma possível o dia de operação do aeroporto. Além das operações diárias, corretamente distribuídas entre os tipos de aeronaves a serem simuladas, é necessário também definir quais os procedimentos utilizados para pousos e decolagens.

Nos dois estudos propostos serão mantidos os procedimentos padrões das aeronaves para a operação total do aeroporto para pousos e decolagens nas duas cabeceiras da pista (Cab. 18 Norte Magnético e Cab. 36 - Sul Magnético) das aeronaves do período.

Conforme as cartas do Departamento de Controle do Espaço Aéreo - DECEA (2010) existem, para o aeroporto de Ribeirão Preto (SBRP), 6 procedimentos padrão de decolagem (ALVA, BOFE, FRANCA, LAMU, MARA, ULAM), e dois de pouso RNAV (GNSS) e NDB (nondirectional beacon) NORTE e SUL para as duas cabeceiras. 


\section{Análise e resultado}

As Figuras 3 e 4 apresentam curvas de ruído, de 10 em 10 dB(A), simuladas pelo INM e sobrepostas sobre o mapa da cidade de Ribeirão Preto com o programa TransCAD for Windows.

A Tabela 1 apresenta a latitude e longitude das cabeceiras da pista obtidas por GPS relativo ao Marco ICA em decimal. As cotas foram obtidas com estação total partindo do marco de referencia do aeroporto em metros.

Tabela 1 - Latitude e Longitude das cabeceiras e cotas $\left(^{1}\right)$

\begin{tabular}{ccccccc}
\hline Ponto & Latitude & Longitude & Distancia $(\mathrm{m})$ & Ângulo & Cota $(\mathrm{m})$ & Cota $(\mathrm{ft})$ \\
\hline Cab 18 & 21,125099 & 47,777035 & 1599,33 & $213^{\circ} 48^{\prime} 57^{\prime \prime}$ & 548,08 & 1798 \\
\hline 2Cab Rec & 21,12682 & 47,776481 & 1401,22 & $214^{\circ} 52^{\prime} 02^{\prime \prime}$ & 547,04 & 1795 \\
\hline Cab 36 & 21,14325 & 47,77122 & 552,95 & $04^{\circ} 37^{\prime} 06^{\prime \prime}$ & 550,31 & 185 \\
\hline Cab 36 Rec & 21,142385 & 47,771498 & 461,54 & 0 & 550,02 & 1805 \\
\hline Ref SC & 21,139344 & 47,774537 & 0 & 0 & 549,23 & 1802 \\
\hline
\end{tabular}

Fazendo uso dos dados obtidos no censo do IBGE (CENSO, 2000) da cidade de Ribeirão Preto (Figura 1) é possível verificar através de "ID" do código de setor (identificação interna da tabela do IBGE) a população residente nestes setores. A Tabela 2 apresenta o número de habitantes em cada setor que será utilizado na simulação do INM para se estimar o total dentro de cada curva de ruído mostrada nas Figuras 3 e 4.

\footnotetext{
${ }^{1}$ Fonte: Autores
} 


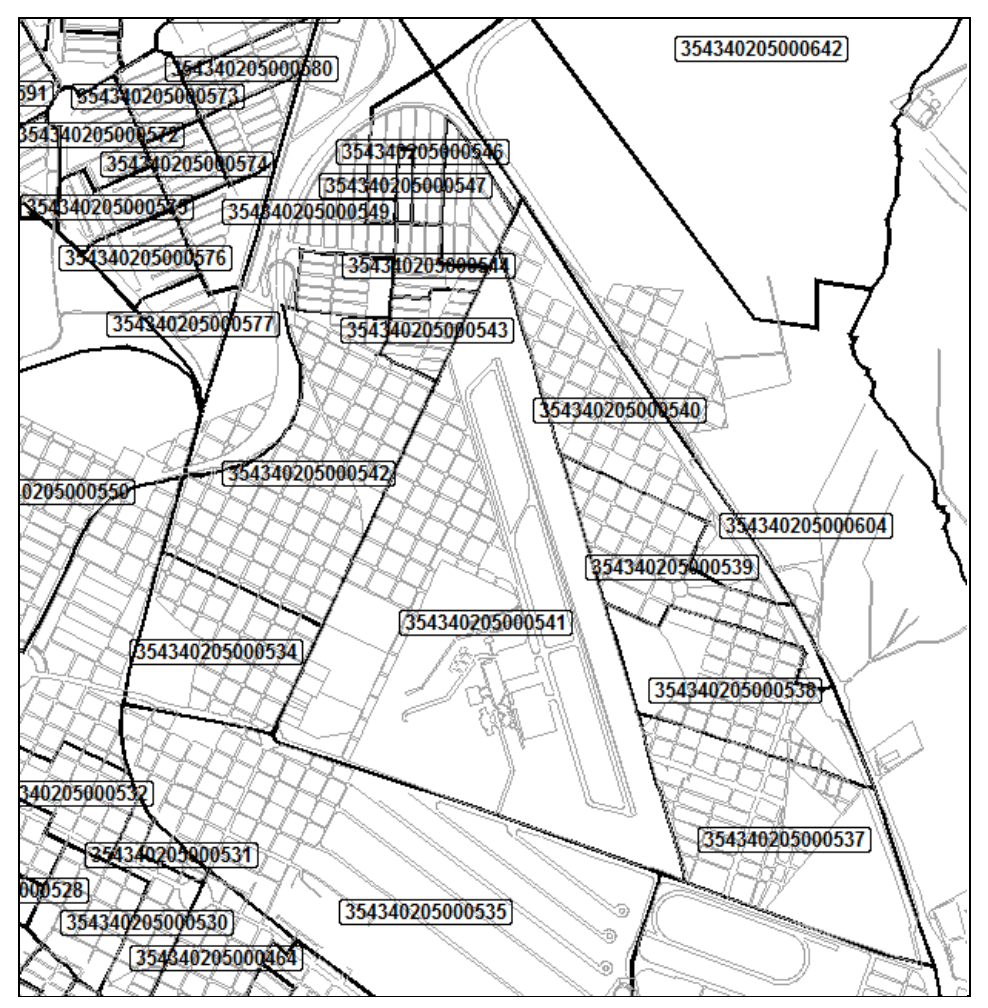

Figura 1 - Identificação dos setores censitários com os dados do IBGE

Tabela 2 - Dados do IBGE (2000) da cidade de Ribeirão Preto $\left(^{3}\right)$

\begin{tabular}{cccc}
\hline \multicolumn{5}{c}{ Tabela de valores por Setor da variável } \\
\hline \multicolumn{4}{c}{ Pessoas residentes } \\
\hline Código Setor & ID & Nome Município & Habitante \\
\hline 354340205000534 & 534 & Ribeirão Preto & 785 \\
\hline 354340205000535 & 535 & Ribeirão Preto & 934 \\
\hline 354340205000536 & 536 & Ribeirão Preto & 1937 \\
\hline 354340205000537 & 537 & Ribeirão Preto & 1253 \\
\hline 354340205000538 & 538 & Ribeirão Preto & 2666 \\
\hline 354340205000539 & 539 & Ribeirão Preto & 2043 \\
\hline 354340205000540 & 540 & Ribeirão Preto & 1508 \\
\hline 354340205000541 & 541 & Ribeirão Preto & 547 \\
\hline 354340205000542 & 542 & Ribeirão Preto & 703 \\
\hline 354340205000543 & 543 & Ribeirão Preto & 1082 \\
\hline 354340205000544 & 544 & Ribeirão Preto & 811 \\
\hline 354340205000545 & 545 & Ribeirão Preto & 697 \\
\hline 354340205000546 & 546 & Ribeirão Preto & 1033 \\
\hline 354340205000547 & 547 & Ribeirão Preto & 636 \\
\hline 354340205000548 & 548 & Ribeirão Preto & 1175 \\
\hline 354340205000549 & 549 & Ribeirão Preto & 990 \\
\hline
\end{tabular}

${ }^{3}$ Fonte: Autores 
A Figura 2 mostra a localização dos pontos, cujas coordenadas foram determinadas com receptor GPS Garmin 60CSx, onde foram feitas as leituras com medidores de nível de pressão sonora.

A Tabela 3 foi elaborada com os dados obtidos da simulação e do levantamento de campo. Estes pontos foram retirados da simulação e suas coordenadas foram locadas no campo com uma estação total onde após, estes pontos foram monitorados com dois decibelimetros instalados paralelos ao eixo da pista e assim foram observadas todas as operações relevantes das aeronaves de maior ruído. Entre estas observações estavam os B737-300 que era o objeto do nosso estudo.

Foi utilizado o marco ICA como referencia para a implantação dos pontos do levantamento de campo.

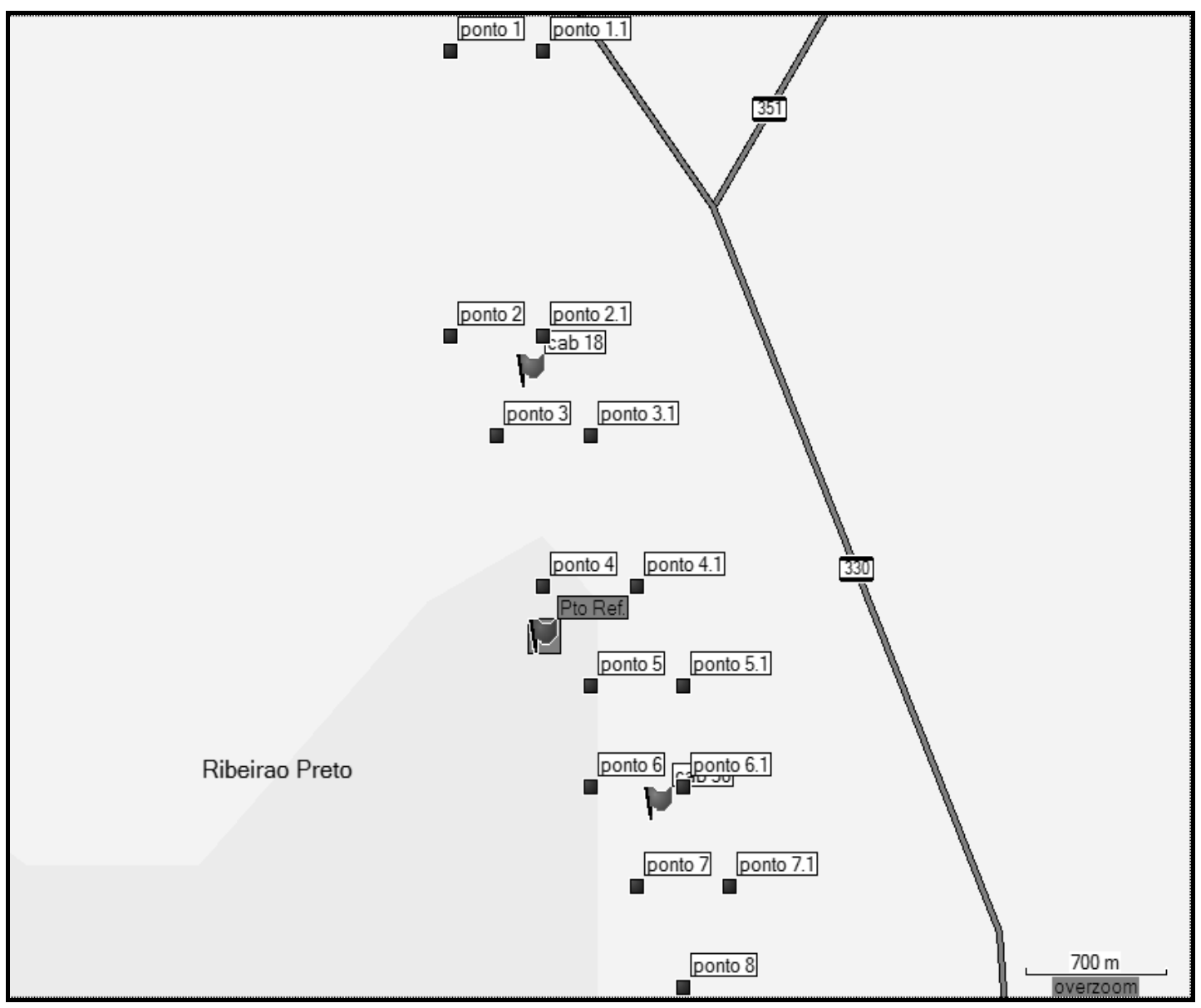

Figura 2 - Localização dos pontos de coleta de dados elaborado pelo MapSource Trip \& Waypoint Manager 
Tabela 3 - Planilha de dados elaborada pelo autor dos pontos levantados

\begin{tabular}{|c|c|c|c|c|c|c|c|c|c|c|}
\hline PONTO & LATITUDE & LONGITUDE & LATITUDE - GPS & LONGITUDE - GPS & DISTANCIA & ÂNGULO & $\mathrm{d}(\mathrm{B}) \mathrm{A}-\mathrm{INM}$ & $\mathrm{d}(\mathrm{B}) \mathrm{A}-\mathrm{Campo}$ & $\operatorname{COTA}(\mathrm{m})$ & $\operatorname{COTA}(\mathrm{ft})$ \\
\hline cab18 & 21,125099 & 47,777035 & 21,125099 & 47,777035 & 1599,33 & $213^{\circ} 48^{\prime} 57^{\prime \prime}$ & & & 548,08 & 1798,16 \\
\hline cab18rec & 21,126820 & 47,776481 & 21,126820 & 47,776481 & 1401,22 & $214^{\circ} 52^{\prime} 02^{\prime \prime}$ & & & 547,04 & 1794,75 \\
\hline cab36 & 21,143250 & 47,771220 & 21,143250 & 47,771220 & 552,95 & $04^{\circ} 37^{\prime} 06^{\prime \prime}$ & & & 550,31 & 1805,47 \\
\hline cab36rec & 21,142385 & 47,771498 & 21,142385 & 47,771498 & 461,54 & 0 & & & 550,02 & 1804,52 \\
\hline ref sc & 21,139344 & 47,774537 & 21,139344 & 47,774537 & 0 & 0 & 64,7 & 90,87 & 549,23 & 1801,93 \\
\hline P 2 & 21,124708 & 47,778992 & 21,12591 & 47,77863 & 1685,23 & $207^{\circ} 52^{\prime} 56^{\prime \prime}$ & 67,7 & 86,79 & 545,60 & 1790,02 \\
\hline P 2.1 & 21,124708 & 47,776764 & 21,12473 & 47,77696 & 1636,86 & $215^{\circ} 00^{\prime} 19^{\prime \prime}$ & 80,7 & 91,59 & 546,31 & 1792,35 \\
\hline P 3.3 & 21,128889 & 47,776764 & 21,128889 & 47,776764 & 673,73 & $207^{\circ} 58^{\prime} 12^{\prime \prime}$ & 74,3 & 85,64 & 546,77 & 1793,86 \\
\hline P 3.1 & 21,128889 & 47,774536 & 21,12889 & 47,77468 & 1157,51 & $223^{\circ} 08^{\prime} 04^{\prime \prime}$ & 70,4 & & 545,53 & 1789,79 \\
\hline $\mathrm{P} 4$ & 21,135162 & 47,772308 & 21,135162 & 47,772308 & 466,21 & $216^{\circ} 21^{\prime} 53^{\prime \prime}$ & 66,5 & 80,94 & 546,34 & 1792,45 \\
\hline P 4.1 & 21,135162 & 47,775065 & 21,13518 & 47,77238 & 517,62 & $249^{\circ} 41^{\prime} 51^{\prime \prime}$ & 67 & & 543,15 & 1781,98 \\
\hline $\mathrm{P} 5$ & 21,139343 & 47,774536 & 21,13931 & 47,77456 & 0 & 0 & 64,7 & 90,87 & 549,23 & 1801,93 \\
\hline $\mathrm{P} 5.1$ & 21,139343 & 47,770882 & 21,13931 & 47,77098 & 379,61 & $313^{\circ} 08^{\prime} 07^{\prime \prime}$ & 64 & 79,64 & 545,47 & 1789,60 \\
\hline P 6 & 21,143526 & 47,774536 & 21,14351 & 47,7745 & 463,14 & $43^{\circ} 08^{\prime} 4^{\prime \prime}$ & 60,6 & 71,66 & 551,74 & 1810,17 \\
\hline P 6.1 & 21,143526 & 47,770081 & 21,14346 & 47,77002 & 654,7 & $358^{\circ} 09^{\prime} 33^{\prime \prime}$ & 71 & 82,93 & 548,20 & 1798,55 \\
\hline
\end{tabular}


A Figura 3 apresenta as curvas de nível de ruído projetadas na malha viária da cidade antes, em azul, e depois da inclusão dos novos voos, em vermelho, para as mesmas condições ambientais (temperatura $15^{\circ} \mathrm{C}$ e vento $0 \mathrm{kt}$ ). Fica bem claro o acréscimo da extensão da área e consequentemente da população afetada, onde a população a Oeste e na direção Sul do eixo da pista recebem maior impacto sonoro com a introdução dos novos voos.

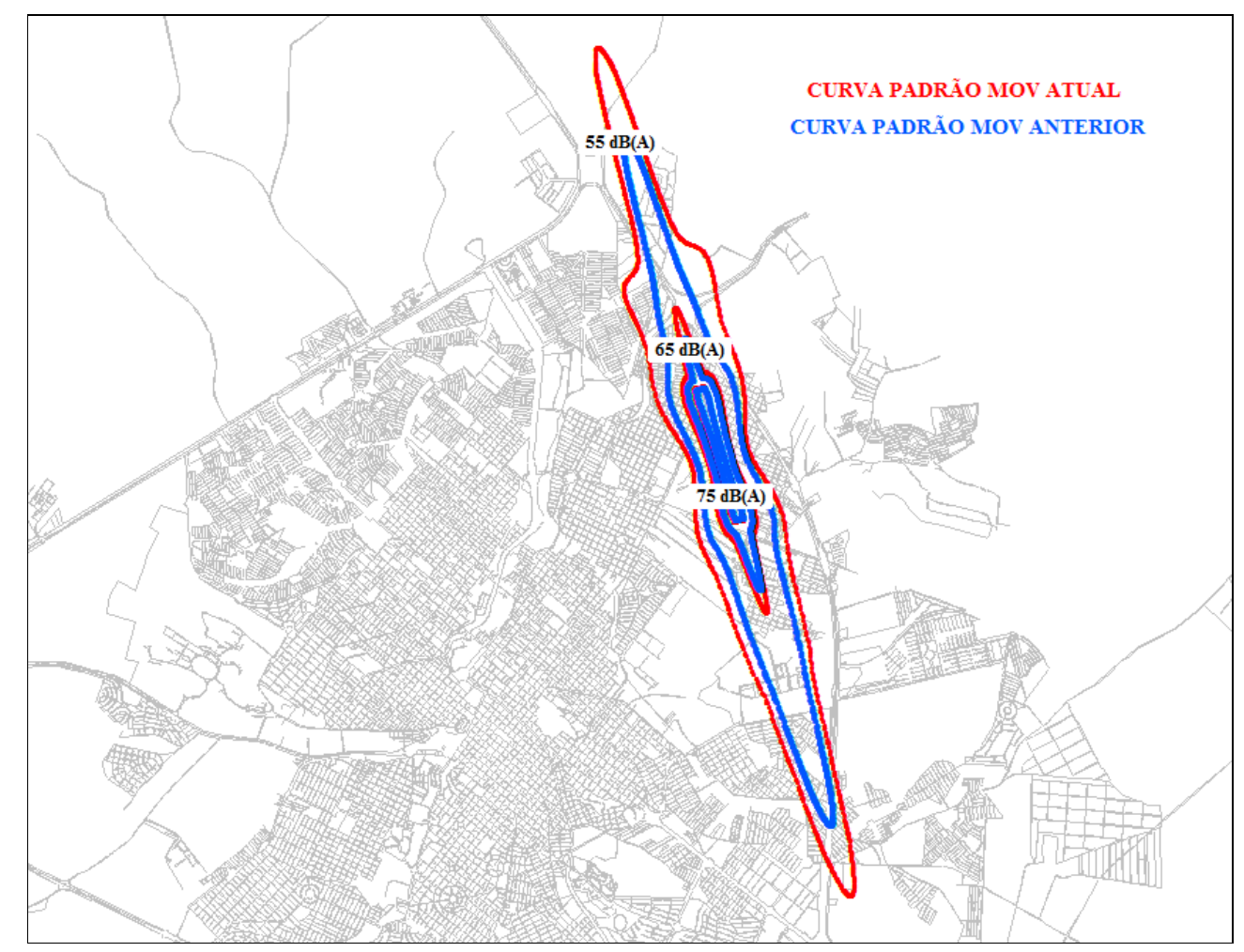

Figura 3 - Curvas de ruído total na forma padrão em $L_{d n}\left({ }^{4}\right)$

A Tabela 4 apresenta para cada faixa de nível de ruído a quantidade de população afetada no movimento anterior das aeronaves e no atual e as respectivas áreas.

Tabela 4 - Resultados da simulação obtidos pelo INM na Figura $5\left(^{5}\right)$

\begin{tabular}{ccccccc}
\hline $\begin{array}{c}\text { Nível } \\
\mathrm{dB}(\mathrm{A})\end{array}$ & $\begin{array}{c}\text { Pop. mov. } \\
\text { anterior }\end{array}$ & $\begin{array}{c}\text { Área } \mathrm{km}^{2} \\
\text { anterior }\end{array}$ & $\begin{array}{c}\text { \% Pop } \\
\text { afetada }\end{array}$ & $\begin{array}{c}\text { Pop. mov. } \\
\text { atual }\end{array}$ & $\begin{array}{c}\text { Área } \mathrm{km}^{2} \\
\text { atual }\end{array}$ & $\begin{array}{c}\% \text { Pop } \\
\text { afetada }\end{array}$ \\
\hline 55 a 65 & 14965 & 7,21 & 99 & 29719 & 12,30 & 71 \\
\hline 65 a 75 & 0 & 1,18 & 100 & 2115 & 1,77 & 50 \\
\hline$>75$ & 0 & 0,27 & 0 & 0 & 0,37 & 38 \\
\hline
\end{tabular}

\footnotetext{
${ }^{4}$ Fonte: Autores

${ }^{5}$ Fonte: Autores
} 
A Figura 4 mostra o acréscimo da extensão da área e também da população afetada com a simulação da curva de ruído introduzindo novos voos e operando com temperatura mais elevada e da velocidade do vento acima da normal.

Os procedimentos foram alterados, porém com os mesmos dados da simulação anterior, com a mudança da temperatura que passou para $30^{\circ} \mathrm{C}$ e da velocidade dos ventos para $13 \mathrm{kt}$ (24 $\mathrm{km} / \mathrm{h}$ ). Os parâmetros: peso da aeronave, rotas de pouso e decolagem e rampas de aproximação, permaneceram os mesmos para as duas simulações.

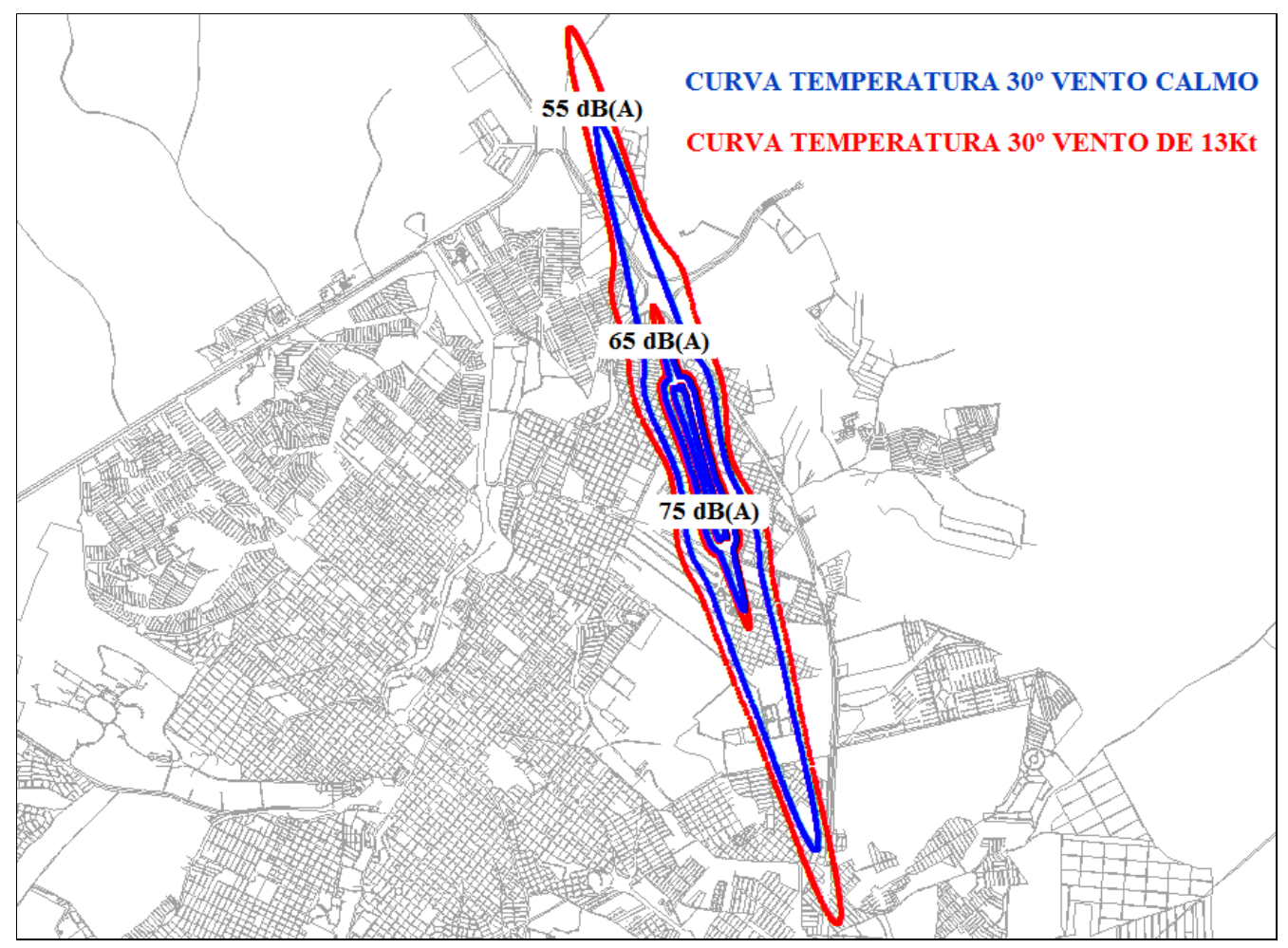

Figura 4 - Curvas de ruído total $30^{\circ} \mathrm{C}$ vento calmo e de 13kt em $L_{d n}\left(^{6}\right)$

A Tabela 5, similarmente a Tabela 4, apresenta os resultados nas novas condições climáticas. Observa-se que com a mudança das condições há uma variação sensível nas porcentagens da população e da área.

\footnotetext{
${ }^{6}$ Fonte: Autores
} 


\section{Tabela 5 - Dados obtidos pelo INM na simulação da Figura $6\left({ }^{7}\right)$}

\begin{tabular}{ccccccc}
\hline $\begin{array}{c}\text { Nível } \\
\mathrm{dB}(\mathrm{A})\end{array}$ & $\begin{array}{c}\text { Pop } 30^{\circ} \mathrm{C} \\
\text { padrão }\end{array}$ & $\begin{array}{c}\text { Área } \\
\mathrm{km}^{2}\end{array}$ & $\begin{array}{c}\text { Pop } \\
30^{\circ} \mathrm{C} 13 \mathrm{kt}\end{array}$ & $\begin{array}{c}\text { Área } \\
\mathrm{km}^{2}\end{array}$ & $\begin{array}{c}\% \text { Pop } \\
\text { afetada }\end{array}$ & $\begin{array}{c}\% \mathrm{~km}^{2} \\
\text { acréscimo }\end{array}$ \\
\hline 55 a 65 & 14418 & 13,42 & 25844 & 21,27 & 79 & 58 \\
\hline 65 a 75 & 0 & 2,47 & 1033 & 3,47 & 100 & 40 \\
\hline$>75$ & 0 & 0,23 & 0 & 0,32 & 0 & 37 \\
\hline
\end{tabular}

\section{Conclusão}

Se espera que exista uma discrepancia nos valores obtidos na simulação e a realidade no campo, mas não se devem desprezar os seus resultados, ou seja, é uma ferramenta que pode ser aplicada para se ter uma ordem de grandeza do impacto causado na circunvizinhança do aeroporto. Estes valores absolutos podem ser verificados na Tabela 3.

Mesmo as medidas de campo, serem diferentes do modelo, como mostra na tabela 3, a sua aplicação é valida em casos que não se possa dispor de medidas de campo.

As discrepâncias podem ser causadas pelo não seguimento dos padrões estabelecidos nas cartas de voo pelo piloto em comando, ventos de direções diversas e não inclusão do relevo da região.

Uma grande vantagem na utilização do INM é que ele é flexível no sentido de que pode facilmente acomodar refinamentos para a mudança das rotas das aeronaves.

Resultante desta simulação pode-se citar algumas soluções sem custo adicional para a administração aeroportuária, para evitar reclamações por parte dos residentes no entorno deste aeroporto, porém estas mudanças só poderão ser executadas com o aval do DECEA e através de estudos mais complexos.

- Inverter a operação das aeronaves passando a utilizar a cabeceira 36 para pousos e decolagens. Isso pode ser feito porque os registros históricos obtidos do Estudo de impacto ambiental do Aeroporto Leite Lopes do mapeamento dos dados meteorológicos indicam que como os ventos de través (leste para oeste) são de baixa velocidade, pode-se considerar essa opção.

\footnotetext{
${ }^{7}$ Fonte: Autores
} 
- Como o pátio de manobras das aeronaves fica mais próximo a cabeceira 36 existirá um ganho considerável no tempo de decolagem e no pouso utilizando a pista de táxi e com isso, liberar mais rapidamente a pista principal.

\section{Referências}

Associação brasileira de normas técnicas - NBR 11415 (1990) Ruído aeronáutico.

Berglund, B., Lindvall, T. e Schwela, D. H. (1999) Guidelines for community noise: Prepared for the World Health Organization, London.

Caliper (2007) TransCAD transportation GIS software: User's guide. Version 4.8. Massachusetts: Academic License, Newton.

Damião, D. P. e Rocha, A. (1998) Utilização de dados de sensoriamento remoto no planejamento urbano do entorno de aeródromos: Anais IX Simpósio brasileiro de sensoriamento remoto, Santos.

Dani, A., Garavelli, S. L. (2001) Principais Efeitos da Poluição Sonora em Seres Humanos. Revista Universa, vol. 9, n. 14, pp. 659-678.

Departamento de Controle do Espaço Aéreo - DECEA (2010), Sítio da internet, disponível em Www.decea.gov.br.

Federal Aviation Administration (FAA) (2004) Part 150 Noise compatibility program.

Federal Aviation Administration (FAA) (1983) Noise control and compatibility planning for airports. Advisory circular AC150-5020-1, U. S. Department of transportation, Washington.

Fernandes, J. C. (2002) Acústica e ruído. Apostila do Curso de Especialização em Engenharia de Segurança do Trabalho. UNESP - Campus de Bauru.

Figueiredo Ferraz Consultoria e Engenharia de Projetos Ltda (2005) Estudo de impacto ambiental Aeroporto Leite Lopes. Documento técnico, São Paulo.

Horonjeff, R and McKelvey, F. (1993) Planning and a design of airports. Forth edition, McGrawHill.

Instituto Brasileiro de Geografia e Estatística - IBGE (2000) Censo Demográfico 2000. Rio de Janeiro: IBGE.

ICAO - CAN (1970) Committee on aircraft noise.

ICAO (1993) Environmental protection, Annex 16, volume 1: aircraft noise, Third edition, Montreal.

ICAO (2001) Apêndice C da A33-7: Consolidated statement of continuing ICAO policies and practices related to environmental protection, pp. 15-17.

ICAO (2002) Airport planning manual - Land use and environmental control DOC 9184 AN/902 Part 2, Third edition, Montreal.

ICAO (2004) Environmental technical manual on the use of procedures in the noise certification of aircraft Doc 9501 AN/929, Third Edition. Quebec.

Macedo, M. R. V. (2004) Avaliação pós-ocupação acústica: um instrumento de apoio ao planejamento e gestão ambiental na circunvizinhança de pequenos aeroportos. Tese (Doutorado em Engenharia de Produção), Universidade Federal do Rio de Janeiro, Rio de Janeiro. 
Nogueira, R. C. C. e Slama, J. G., (2005) Implementing the balanced approach for airport noise control in developing countries: the case of Brazil. Proceedings of the Internoise, Rio de Janeiro.

Olmstead, J. R., Fleming, G. G., Gulding, J. M., Roof, C. J., Gerbi, P. J. e Rapoza, A. S. (2002) Integrated noise model (INM) Version 6.0 Technical manual, Report FAA-AEE-02- 01, Office of environment and energy, Federal Aviation Administration.

Revoredo, T. C. (2011) Estimação dinâmica do ruído aeronáutico utilizando sistemas diferencialmente planos e redes neurais artificiais. Tese (Doutorado em Engenharia Mecânica),Universidade Federal do Rio de Janeiro. Rio de Janeiro.

Society of Automotive Engineers (1986) SAE AIR 1845. Procedure for calculation of airplane noise in the vicinity of airports. Warrendale.

Vallet, M. (2002) Acoustic pollution due to aircraft traffic and the ways to reduce it. Euronoise, Madrid.

Werlich, J. M. and Krinsky, R. P. (1981) The aviation noise abatement controversy: Magnificent laws, noisy machines, and the legal liability shuffle, Loyola of Los Angeles Law Review, vol. 15.

World Health Organization (WHO) (2010) International society of hypertension guidelines for the management of hypertension. Guidelines Subcommittee.

World Health Organization (WHO) (1999) Guide lines for community noise. London, Stockholm University and Karolinska Institute. 